\title{
Correction to: A Review of Interventions to Enhance HIV Medication Adherence
}

\author{
Laura B. Whiteley $^{1}$ (D) Elizabeth M. Olsen ${ }^{1} \cdot$ Kayla K. Haubrick $^{2} \cdot$ Enyonam Odoom $^{2} \cdot$ Nicholas Tarantino $^{2}$. \\ Larry K. Brown ${ }^{2}$
}

Published online: 15 September 2021

(c) Springer Science+Business Media, LLC, part of Springer Nature 2021

\section{Correction to: Current HIV/AIDS Reports \\ https://doi.org/10.1007/s11904-021-00568-9}

The original version of this review article unfortunately contained a mistake in the Abstract section. The revised version is presented below:

\begin{abstract}
Purpose of Review Adherence to antiretroviral treatment (ART) is crucial for the successful treatment of HIV. Unfortunately, it is estimated that $45 \%$ of persons living with HIV (PLWH) have poor adherence to ART. To provide health care professionals and PLWH with effective tools for supporting adherence, researchers have investigated the effectiveness of psychosocial interventions to enhance adherence to ART. In this paper, interventional studies, systematic reviews, and meta-analyses that examine ART adherence interventions for PLWH are reviewed.

Recent Findings There is great variability among interventions in terms of quality, sample, measures, and outcome characteristics. Despite a diverse and wide-ranging assortment of ART adherence interventions, consistent lessons have been learned. Interventions that focus on individual and interpersonal factors have been effective for improving ART adherence; however, the improvement in adherence tends to be short-lived. Additionally, interventions are most successful when tailored to those at risk for poor adherence. Finally, theory-based interventions are more likely to be effective than non-theory-based interventions.

Summary A variety of individual-level psychological interventions have been shown to be effective in improving ART adherence in the short term. Digital and mobile interventions have the potential to improve dissemination and implementation of these evidence-based interventions and could be used to extend intervention effects. Future interventions that address issues of accessibility, inequality, structural and institutional barriers to ART adherence should also be tested and prioritized. Implementation science frameworks can be used to assess and address issues of accessibility and systematic barriers to care.
\end{abstract}

The original article has been corrected.

Publisher's Note Springer Nature remains neutral with regard to jurisdictional claims in published maps and institutional affiliations.

The original article can be found online at https://doi.org/10.1007/

s11904-021-00568-9.

Laura B. Whiteley

Laura_Whiteley@brown.edu

1 Department of Psychiatry and Human Behavior, The Warren Alpert Medical School of Brown University, Providence, RI, USA

2 Department of Psychiatry, Rhode Island Hospital, Providence, RI, USA 